\title{
Generalized uncertainty principle and corpuscular gravity
}

\author{
Luca Buoninfante $^{1,2,3, \mathrm{a}} \mathbb{D}_{\mathrm{D}}$, Giuseppe Gaetano Luciano ${ }^{1, \mathrm{~b}}$, Luciano Petruzziello ${ }^{1,2, \mathrm{c}}$ \\ ${ }^{1}$ INFN, Sezione di Napoli, Gruppo collegato di Salerno, 84084 Fisciano, SA, Italy \\ 2 Dipartimento di Fisica, Università di Salerno, Via Giovanni Paolo II, 132, 84084 Fisciano, SA, Italy \\ ${ }^{3}$ Van Swinderen Institute, University of Groningen, 9747 AG Groningen, The Netherlands
}

Received: 12 June 2019 / Accepted: 23 July 2019 / Published online: 7 August 2019

(C) The Author(s) 2019

\begin{abstract}
We show that the implications of the generalized uncertainty principle (GUP) in the black hole physics are consistent with the predictions of the corpuscular theory of gravity, in which a black hole is conceived as a BoseEinstein condensate of weakly interacting gravitons stuck at the critical point of a quantum phase transition. In particular, we compute such characteristic thermodynamic quantities as the temperature and the evaporation rate of a black hole. By comparing the results obtained in the two scenarios, we are able to estimate the GUP deformation parameter $\beta$, which turns out to be of order unity, in agreement with the expectations of some models of string theory. We also comment on the sign of $\beta$, exploring the possibility of having a negative deformation parameter when a corpuscular quantum description of the gravitational interaction is assumed to be valid.
\end{abstract}

\section{Introduction}

One of the most difficult and stimulating challenges the physics community has been struggling with for a long time is to understand whether the gravitational interaction has an intrinsic quantum nature and, if so, how to formulate a thorough quantum theory of gravity which avoids conceptual problems and is able to make successful predictions at any energy scale. In pursuing the aim of combining both gravitational and quantum effects, the question inevitably arises as to whether the basic principles of quantum mechanics need to be revised in the quantum gravity realm.

\footnotetext{
a e-mail: lbuoninfante@sa.infn.it

b e-mail: gluciano@sa.infn.it

c e-mail: lpetruzziello@na.infn.it
}

It is well known that one of the fundaments of quantum mechanics is the Heisenberg Uncertainty Principle (HUP) ${ }^{1}$ [1],

$\delta x \delta p \geq \frac{\hbar}{2}$,

which can be derived from the non-vanishing commutator between the position and momentum operators $\hat{x}$ and $\hat{p}$, respectively,

$[\hat{x}, \hat{p}]=i \hbar$.

The above inequality asserts that, in the quantum regime, the more precisely the position (momentum) of a particle is known, the less precisely one can say what its momentum (position) is. This implies the existence of a region $\delta x \delta p$ of size $\hbar$ in the phase space, in which any physical prediction cannot be tested. In spite of this, however, no quantum limit on sharp measurements of either position or momentum separately is fixed a priori: in other terms, arbitrarily short distances may in principle be detected via arbitrarily high energy probes, and vice-versa.

The situation becomes more subtle if one tries to merge quantum and gravity effects within the same framework. In that case, indeed, several models of quantum gravity propose the existence of a minimum length at Planck scale [2] that accounts for a limited resolution of spacetime. The Planck length $\ell_{p}$ thus appears as a natural threshold beyond which spacetime would no longer be smooth, but rather it would have a foamy structure due to inherent quantum fluctuations [3].

Along this line, many studies [4-27] have converged on the idea that the HUP should be properly modified at the

\footnotetext{
${ }_{1}^{1}$ Throughout the paper, we work with the units $c=k_{\mathrm{B}}=1$, where $k_{\mathrm{B}}$ is the Boltzmann constant, and $\hbar \neq 1$. The Planck length is defined as $\ell_{p}=\sqrt{\hbar G}$, while the Planck mass as $m_{p}=\hbar / \ell_{p}$.
} 
quantum gravity scale, in order to accommodate the existence of such a fundamental length. In this sense, one of the most adopted generalizations of the uncertainty principle (GUP) reads

$\delta x \delta p \geq \frac{\hbar}{2} \pm 2|\beta| \ell_{p}^{2} \frac{\delta p^{2}}{\hbar}=\frac{\hbar}{2} \pm 2|\beta| \hbar \frac{\delta p^{2}}{m_{p}^{2}}$,

where the sign \pm refers to positive/negative values of the dimensionless deformation parameter $\beta$, which is assumed to be of order unity in some models of quantum gravity, and in particular in string theory $[8,10]$. However, one could also investigate Eq. (3) from a phenomenological point of view, and seek experimental bounds on $\beta$ (for a recent review of the various phenomenological approaches, see, for example, Ref. [28]). Clearly, for $\beta \hbar / m_{p}^{2} \rightarrow 0$, standard quantum mechanics is recovered, so that modifications to the HUP only become relevant at the Planck scale, as expected. Moreover, for mirror-symmetric states (i.e. $\langle\hat{p}\rangle=0$ ), Eq. (3) can be deduced from the modified commutator

$[\hat{x}, \hat{p}]=i \hbar\left(1 \pm|\beta|\left(\frac{\hat{p}^{2}}{m_{p}^{2}}\right)\right)$.

If, on the one hand, the assumption that $\beta \sim \mathscr{O}(1)$ is quite generally accepted and it has also been confirmed by achievements in contexts other than string theory $[15,22-$ $24,26]$, on the other hand the problem of the sign of $\beta$ is much more debated. Although in various derivations and gedanken experiments on GUP it seems more reasonable to have a positive parameter, arguments in favor of the opposite choice are not lacking. For instance, in Ref. [29] it was emphasized that the GUP with $\beta<0$ would be consistent with a description in which the universe has an underlying crystal lattice-like structure. Similarly, in Ref. [30] a negative $\beta$ was proved to be compatible with the Chandrasekhar limit for white dwarfs. ${ }^{2}$ Further confirmations were given starting from a non-commutative Schwarzschild geometry [28], or a deformed static Schwarzschild metric [32].

In this connection, a clue to an answer for such a dichotomy may be found within the framework of Corpuscular Gravity (CG) originally formulated in Refs. [33,34], and then revisited from a complementary point of view in Refs. [35,36] (see also Refs. [37-43] for further developments). According to this model, black holes can be understood as a Bose-Einstein condensate of weakly interacting gravitons at the critical point of a quantum phase transition. As a consequence of this portrait, black hole characteristics get non-trivially modified.

${ }^{2}$ It must be pointed out, however, that in Ref. [31] it was argued that this issue can be fixed also for the case $\beta>0$, provided that a non-vanishing cosmological constant is included in the treatment.
Starting from the outlined scenario, in the present paper we provide a link between the GUP black hole thermodynamics and the corpuscular model of gravity. Specifically, by comparing the evaporation rate computed via the two different approaches, we infer that they are consistent, provided that $\beta$ is of order unity, in agreement with some models of string theory. We further comment on the sign of $\beta$, speculating on the possibility to obtain a negative value.

The paper is organized as follows: Sect. 2 is devoted to a review of black hole thermodynamics in the context of the GUP. We compute corrections to the Hawking temperature and the evaporation rate, both for positive and negative values of the deformation parameter $\beta$. In Sect. 3 we discuss some fundamental aspects of $\mathrm{CG}$, focusing on the derivation of the formula for the emission rate. GUP and CG approaches are then compared in Sect. 4. By requiring that the modified expressions of the evaporation rate derived in the two frameworks are equal (at the leading order), we are able to evaluate the GUP parameter $\beta$. Finally, conclusions can be found in Sect. 5.

\section{Uncertainty relations and black hole thermodynamics}

Let us consider a spherically symmetric black hole with mass $M$ and Schwarzschild radius $r_{s}=2 M G$. Following the arguments of Refs. $[13,19]$, the Hawking temperature $T_{\mathrm{H}}$ can be derived in a heuristic way by using the HUP (1) and general properties of black holes. To this aim, let us observe that, just outside the event horizon, the position uncertainty of photons emitted by the black hole is of the order of its Schwarzschild radius, i.e. $\delta x \simeq \mu r_{s}$, where the constant $\mu$ is of order of unity and will be fixed below. From Eq. (1), the corresponding momentum uncertainty is given by

$\delta p \simeq \frac{\hbar}{4 \mu M G}$

which also represents the characteristic energy of the emitted photons, since $\delta p \simeq p=E$. According to the equipartition theorem, this can be now identified with the temperature $T$ of the ensemble of photons,

$E=T \simeq \frac{\hbar}{4 \mu M G}=\frac{m_{p}^{2}}{4 \mu M}$

which agrees with the Hawking temperature,

$T_{\mathrm{H}}=\frac{\hbar}{8 \pi M G} \equiv \frac{m_{p}^{2}}{8 \pi M}$,

provided that $\mu=2 \pi$.

Therefore, on the basis of the HUP and thermodynamic consistency, we have recovered the standard Hawking for- 
mula (7) for the temperature of the radiation emitted by the black hole.

Now, it is well known that black holes with temperature greater than the background temperature (about $2.7 \mathrm{~K}$ for the present universe) shrink over time by radiating energy in the form of photons and other ordinary particles. In certain conditions [44], however, it is reasonable to assume that the evaporation is dominated by photon emission. In this case, we can exploit the Stefan-Boltzmann law to estimate the radiated power $P$ as

$P=A_{s} \varepsilon \sigma T^{4} \simeq A_{s} \sigma T^{4}$

where $A_{s}=4 \pi r_{s}^{2}$ is the black hole sphere surface area at Schwarzschild radius $r_{s}, \sigma=\pi^{2} / 60 \hbar^{3}$ is the StefanBoltzmann constant, and we have assumed for simplicity the black hole to be a perfect blackbody, i.e. $\varepsilon \simeq 1$.

Using Eqs. (7) and (8), the black hole energy loss can be easily evaluated as a function of time, yielding

$$
\begin{aligned}
\frac{d M}{d t} & =-P \simeq-\frac{1}{60(16)^{2} \pi \sqrt{\hbar G}} \frac{m_{p}^{3}}{M^{2}} \\
& =-\frac{1}{60(16)^{2} \pi} \frac{m_{p}^{4}}{\hbar M^{2}} .
\end{aligned}
$$

Therefore, the evaporation process leads black holes to vanish entirely with both the temperature (6) and emission rate (9) blowing up as the mass decreases.

The above results have been derived starting from the HUP (1). Let us now perform similar calculations by resorting to the GUP in Eq. (3), so as to realize to what extent the GUP affects the black hole thermodynamics.

In this case, solving Eq. (3) with respect to the momentum uncertainty $\delta p$ and setting again $\delta x$ of the order of the Schwarzschild radius, we obtain the following expression for the modified Hawking temperature

$T_{\mathrm{GUP}}= \pm \frac{M}{4 \pi|\beta|}\left(1 \pm \sqrt{1 \mp|\beta| \frac{m_{p}^{2}}{M^{2}}}\right)$

In the semiclassical limit $\sqrt{|\beta|} m_{p} / M \ll 1$, this agrees with the standard Hawking result in Eq. (6), provided that the negative sign in front of the square root is chosen, whereas the positive sign has no physical meaning. Similarly, the emission rate in Eq. (9) is modified as

$$
\left(\frac{d M}{d t}\right)_{\mathrm{GUP}} \simeq \frac{-1}{60(16) \pi \hbar} \frac{M^{6}}{|\beta|^{4} m_{p}^{4}}\left(1-\sqrt{1 \mp|\beta| \frac{m_{p}^{2}}{M^{2}}}\right)^{4}
$$

In what follows, the implications of Eqs. (10) and (11) will be discussed separately for the cases of $\beta>0$ and $\beta<0$.

\subsection{GUP with $\beta>0$}

Let us start by analyzing the most common setting of GUP with positive deformation parameter. In this case, from Eq. (10) it is easy to see that the GUP naturally introduces a minimum size allowed for black holes: for $M<\sqrt{\beta} m_{p}$ (i.e. $r_{s}<2 \sqrt{\beta} \ell_{p}$ ), indeed, the temperature would become complex, in contrast with predictions of ordinary black hole thermodynamics. Remarkably, one can verify that the evaporation process should stop at $M \sim \sqrt{\beta} m_{p}$ by observing that the specific heat of the black hole $d M / d T$ is negative under GUP modification and it vanishes as $M \rightarrow \sqrt{\beta} m_{p}$ [45], thus leading to an inert remnant with finite temperature and size. On the other hand, different models predict a change of sign in the specific heat as the mass is wiped out; in that case black hole remnants may undergo a phase transition which renders them unstable. Notice also that the idea of black hole remnants dates back to Aharonov-Casher-Nussinov, who first addressed the issue in the context of the black hole unitarity puzzle [46].

Similarly, concerning the modified emission rate (11), we find that it is finite at the endpoint of black hole evaporation $M \sim \sqrt{\beta} m_{p}$, whereas the corresponding HUP result (9) diverges at the endpoint when $M=0$.

\subsection{GUP with $\beta<0$}

Although the GUP with $\beta>0$ cures the undesired infinite final temperature predicted by Hawking's formula (7), it would create several complications, such as the entropy/information problem $[47,48]$, or the removal of the Chandrasekhar limit [30]. The latter prediction, in particular, would allow white dwarfs to be arbitrarily large, a result that is at odds with astrophysical observations. An elegant way to overcome these ambiguities was proposed in Ref. [30], where it was shown that both the infinities in black hole and white dwarf physics can be avoided by choosing a negative deformation parameter in Eq. (3). A similar scenario had previously been encountered in Ref. [29] in the context of GUP in a crystal-like universe with lattice spacing of the order of Planck length.

Let us then consider the case $\beta<0$. With this setting, from Eqs. (10) and (11) we obtain that both the modified temperature and emission rate are well defined even for $M<\sqrt{|\beta|} m_{p}$. For a sufficiently small $M$, in particular, the modified temperature in Eq. (10) can be approximated as

$T_{\mathrm{GUP}} \simeq \frac{m_{p}}{4 \pi \sqrt{|\beta|}}<\infty$ 
Even though no lower bound on the black hole size arises in this framework, the Hawking temperature remains finite as the black hole evaporates to zero mass. From Eq. (12) we also deduce that the bound on the Hawking temperature is independent of the initial black hole mass.

\section{Corpuscular black holes}

In the Corpuscular Gravity picture (which was first introduced in Refs. [33,34] and then revised from a different perspective in Refs. [35-43]) black holes can be conceived as Bose-Einstein condensates of $N$ interacting and nonpropagating longitudinal gravitons, and thus as intrinsically quantum objects. Let us recall that a Bose-Einstein condensate of attractive bosons and their phase transitions were firstly studied in Ref. [49] in the context of condensed matter physics.

In order to better understand such a black hole quantum's portrait and make a comparison with the GUP predictions, let us review the most relevant properties of the corpuscular theory of gravity.

\subsection{Bose-Einstein condensate of gravitons}

Let us consider a Bose-Einstein condensate of total mass $M$ and radius $R$, which is made up of $N$ weakly interacting gravitons. At low energy, we can define a quantum gravitational self-coupling for each single graviton of wavelength $\lambda$ as follows [33]

$\alpha_{g} \equiv \frac{\hbar G}{\lambda^{2}}=\frac{\ell_{p}^{2}}{\lambda^{2}}$

One of the main features of a Bose-Einstein condensate is that, due to the interaction, its constituents acquire a collective behavior, so that their wavelengths get increasingly larger and their masses smaller; strictly speaking, the constituents become softer bosons. In particular, most of the gravitons composing the gravitational system will have a wavelength of the order $\lambda \sim R$, namely of the order of the size of the system itself. ${ }^{3}$ Hence, similarly to Eq. (13), it is possible to define a collective quantum coupling as

$N \alpha_{g} \equiv N \frac{\hbar G}{\lambda^{2}} \simeq N \frac{\ell_{p}^{2}}{R^{2}}$

\footnotetext{
3 More precisely, the condensate is also made up of gravitons with shorter wavelengths, $\lambda<R$. However, all physical quantities will be mostly determined by those gravitons that possess a wavelength of the order $\lambda \sim R$, since the contribution arising from hard gravitons turns out to be exponentially suppressed and can thus be neglected [33].
}

We now seek the relation that links the total mass of a BoseEinstein condensate and its radius to the number $N$ of quanta composing the system. By performing a standard computation, one can show that the gravitational binding energy of the system is given by

$E_{g} \simeq \frac{G M^{2}}{R}$.

On the other hand, from a purely quantum point of view, the binding energy can be expressed as the sum of the energies associated to each single graviton, i.e. ${ }^{4}$

$E_{g} \simeq N \frac{\hbar}{\lambda} \simeq N \frac{\hbar}{R}$

Therefore, by comparing Eqs. (15) and (16), we obtain

$M \simeq \sqrt{N} m_{p}$,

which also implies for the Schwarzschild radius

$r_{s} \simeq \sqrt{N} \ell_{p}$.

By assuming that the size of the condensate is $R \sim r_{s}$ (i.e. the overall gravitational system is a black hole) and using the expression in Eq. (18) for the Schwarzschild radius, we notice that the collective quantum coupling defined in Eq. (14) is always of order unity in the case of a black hole

$N \alpha_{g} \simeq 1$.

In condensed matter physics, it is well known that the inequality $N \alpha_{g}<1$ corresponds to a phase in which a BoseEinstein condensate is weakly interacting. On the other hand, the equality $N \alpha_{g}=1$ represents a critical point at which a phase transition occurs, thus letting the condensate become strongly interacting, whereas for $N \alpha_{g}>1$ it is possible to observe only a strongly interacting phase [49]. Thus, in this quantum corpuscular picture, a black hole can be defined as a Bose-Einstein condensate of gravitons stuck at the critical point of a quantum phase transition [34].

\subsection{Thermodynamic properties of corpuscular black holes}

We now analyze some thermodynamic aspects of quantum corpuscular black holes, and in particular we show that gravitons can escape from the considered system. Such a phenomenon represents the corpuscular counterpart of the black hole radiation emission [33].

\footnotetext{
${ }^{4}$ Note that, in Eq. (16), we are allowed to employ the usual de Broglie relation because we are considering low energy weakly interacting gravitons with large wavelengths. Quantum gravity effects only emerge as a collective feature of the whole gravitational system.
} 
First of all, we need to compute the probability for a graviton to escape from a gravitational bound state, namely we have to determine the so-called escape energy and escape wavelength of a single graviton. To this aim, observe that, for $N$ weakly interacting quanta composing a condensate of radius $R$ and mass $M$, a quantum gravitational interaction strength can be defined as [33]

$\hbar N \alpha_{g} \equiv \hbar N \frac{L_{p}^{2}}{\lambda^{2}}$

so that each graviton is subject to the following binding potential

$E_{\mathrm{esc}}=\frac{\hbar N \alpha_{g}}{R}$,

which is the threshold to exceed in order to escape. The corresponding escape wavelength is defined as

$\lambda_{\mathrm{esc}}=\frac{\hbar}{E_{\mathrm{esc}}}$.

If we now employ Eqs. (18) and (19) for the case of a black hole, we obtain

$E_{\mathrm{esc}} \simeq \frac{\hbar}{\sqrt{N} \ell_{p}}, \quad \lambda_{\mathrm{esc}} \simeq \sqrt{N} \ell_{p}$.

This means that, although $N$ gravitons of wavelength $\lambda \sim \sqrt{N} \ell_{p}$ can form a gravitational bound state, at the same time a depletion process is present, which is traduced in a leakage of the constituents of the condensate for any $N$. Clearly, this is related to the fact that $\lambda_{\text {esc }}$ coincides with the wavelength of each graviton belonging to the condensate, that is, $\sqrt{N} \ell_{p}$.

In terms of scattering amplitudes, the above picture can be regarded as a $2 \rightarrow 2$ scattering process, in which one of the two gravitons is energetic enough to be able to exceed the threshold given by $E_{\text {esc }}$.

We can also obtain an estimation for the depletion rate $\Gamma$ of such a process. As usual, this should be given by a product involving the squared coupling constant $\alpha_{g}^{2}$, the characteristic energy scale of the process $E_{\mathrm{esc}}$ and a combinatoric factor $N(N-1)$, which can be approximated by $N^{2}$ for a very large number of constituents [33], i.e.

$\Gamma \simeq \alpha_{g}^{2} N^{2} E_{\mathrm{esc}} \simeq \frac{\hbar}{\sqrt{N} \ell_{p}}$.

From the above relation, we can easily obtain the corresponding time scale of the considered process, which is given by $\Delta t=\hbar / \Gamma \simeq \sqrt{N} \ell_{p}$.
On the other hand, Eq. (24) allows us to infer the mass decrease over time of the condensate, i.e.

$\frac{d M}{d t}=-\frac{\Gamma}{\lambda_{\mathrm{esc}}} \simeq-\frac{\hbar}{N \ell_{p}^{2}} \simeq-\frac{m_{p}^{4}}{\hbar M^{2}}$,

which can be cast in terms of the rate of emitted gravitons by use of Eq. (17),

$\frac{d N}{d t} \simeq-\frac{1}{\sqrt{N} \ell_{p}}$.

We stress that, up to the factor $1 /\left[60(16)^{2} \pi\right]$, Eq. (25) reproduces the thermal evaporation rate of a black hole in Eq. (9), assuming the Hawking temperature in the corpuscular model to be given by [33]

$T_{\mathrm{H}} \simeq \frac{\hbar}{\sqrt{N} \ell_{p}} \simeq \frac{m_{p}^{2}}{M}$

We have seen that the black hole quantum $N$-portrait manages to reproduce the semiclassical result, according to which a black hole emits a thermal radiation with temperature given by the Hawking formula (7). However, from a more scrupulous investigation, one can see that such a result holds true only to the leading order, since in general there will be higherorder corrections which scale as negative powers of the number of gravitons $[33,34,40]$.

In this connection, notice that, in the computation of the depletion rate $\Gamma$ (24), we have only considered the simplest kind of interaction (i.e. a tree-level scattering diagram with two vertices); nevertheless, one expects that even higherorder processes provide $\Gamma$ with contributions that induce gravitons to escape. For instance, the next relevant $2 \rightarrow 2$ scattering process would possess three vertices, thus contributing with terms proportional to $\alpha_{g}^{3}$. Therefore, up to firstorder corrections, the depletion rate would take the form

$$
\begin{aligned}
\Gamma & \simeq \alpha_{g}^{2} N^{2} E_{\mathrm{esc}}+\mathscr{O}\left(\alpha_{g}^{3} N^{2} E_{\mathrm{esc}}\right) \\
& \simeq \frac{\hbar}{\sqrt{N} \ell_{p}}+\mathscr{O}\left(\frac{\hbar}{\ell_{p}} \frac{1}{N^{3 / 2}}\right) .
\end{aligned}
$$

As for the zeroth-order in Eq. (25), the mass decrease of the black hole can be now estimated from the modified depletion rate (28), yielding [34]

$$
\frac{d M}{d t} \simeq-\frac{m_{p}^{4}}{\hbar M^{2}}+\mathscr{O}\left(\frac{m_{p}^{6}}{\hbar M^{4}}\right) .
$$

We emphasize that such a result only describes the qualitative behavior of the evaporation rate in the CG framework. In the next section, we shall consider the exact expression of 
$d M / d t$ in order to make a quantitative comparison with the corresponding GUP result.

\section{Consistency between GUP and corpuscular gravity}

In the previous sections, the evaporation rate of a black hole has been computed within both the GUP and CG frameworks. Here, we compare the two expressions: as it will be shown, this allows us to set the value of the GUP deformation parameter $\beta$ for which the GUP and CG treatments are consistent.

For this purpose, let us consider the GUP-modified expressions of the emission rate (11) expanded up to the order $\mathscr{O}\left(1 / M^{4}\right)$ and the CG outcome (29). We have

$$
\left(\frac{d M}{d t}\right)_{\mathrm{GUP}} \simeq \frac{-1}{60(16)^{2} \pi}\left(\frac{m_{p}^{4}}{\hbar M^{2}} \pm|\beta| \frac{m_{p}^{6}}{\hbar M^{4}}\right)
$$

and

$$
\left(\frac{d M}{d t}\right)_{\mathrm{CG}} \simeq \frac{-1}{60(16)^{2} \pi}\left[\frac{m_{p}^{4}}{\hbar M^{2}}+\mathscr{O}\left(\frac{m_{p}^{6}}{\hbar M^{4}}\right)\right]
$$

where we recall that the sign \pm in Eq. (30) corresponds to a positive/negative value of the deformation parameter $\beta$. Note that, in Eq. (31), the correct numerical factor has been restored by requiring that the Hawking formula (9) is consistently recovered to the zeroth order.

By comparing Eqs. (30) and (31), it follows that, at least up to the first-order, the GUP- and CG-induced corrections exhibit the same functional dependence on the black hole mass. Furthermore, since the coefficient in front of the correction in Eq. (31) is predicted to be of order unity [33,34], numerical consistency between the two expressions automatically leads to

$|\beta| \sim \mathscr{O}(1)$

which is in agreement with the predictions of other models of quantum gravity. Therefore, in spite of their completely different underlying backgrounds, the GUP and CG approaches are found to be compatible with each other.

However, the result (32) does not give any specific information about the sign of $\beta$. Since a full-fledged analytic derivation of Eq. (31) including also higher-order scattering processes is still lacking, a definitive conclusion on this issue cannot be reached. On the one hand, relying on basic considerations on the nature of the scattering amplitudes, we would naively expect the correction in Eq. (31) to contribute with the same sign as the zeroth-order term, since we are only adding higher-order diagrams describing the probability for a graviton to escape from the condensate. This would yield a positive value for the deformation parameter.
On the other hand, there are different claims which assert that the above correction should be opposite to the zerothorder term, in such a way to slightly decrease the evaporation rate of the black hole. This was shown, for example, within the framework of Horizon Quantum Mechanics $[50,51]$, where the depletion rate reads [41]

$\Gamma \simeq \frac{\hbar}{\sqrt{N} \ell_{p}}-\frac{3 \hbar \gamma^{2} N_{H}^{2}}{\ell_{p} N^{3 / 2}}\left(6 \zeta(3)-\frac{\pi^{4}}{15}\right)$

which would imply the following formula for the evaporation rate

$$
\begin{aligned}
\left(\frac{d M}{d t}\right)_{\mathrm{CG}} \simeq & \frac{-1}{60(16)^{2} \pi}\left[\frac{m_{p}^{4}}{\hbar M^{2}}\right. \\
& \left.-3 \gamma^{2} N_{H}^{2}\left(6 \zeta(3)-\frac{\pi^{4}}{15}\right) \frac{m_{p}^{6}}{\hbar M^{4}}\right],
\end{aligned}
$$

where $N_{H} \equiv \sqrt{3} / \sqrt{\pi^{2}-6 \zeta(3)} \simeq 1.06$ and $\zeta(x)$ is the Riemann zeta function. Note also that the constant factor in Eq. (34) is given by $3 \hbar \gamma^{2} N_{H}^{2}\left(6 \zeta(3)-\pi^{4} / 15\right) \simeq 2.4 \gamma^{2}$ and $\gamma$ may be of order one [41]. With such a setting, the comparison of Eqs. (30) and (34) would further confirm the result of Eq. (32), i.e.

$|\beta| \simeq 2.4$

but it would lead to a negative value for the deformation parameter,

$\beta<0$.

Moreover, we remark that positive corrections to the evaporation rate of a black hole are required by the principle of energy conservation [52,53], thus enforcing the validity of Eq. (36). A discussion on the physical meaning and implications of such a result can be found in the Sect. 5 .

\section{Conclusions}

In this paper, we have analyzed to what extent the black hole thermodynamics gets modified both in the presence of a generalized uncertainty principle (GUP) and in the corpuscular gravity (CG) theory. In particular, we have focused on the computation of the temperature and the evaporation rate of a black hole. By comparing the expressions derived within the two frameworks, we have finally managed to estimate the GUP deformation parameter $\beta$. The obtained result shows that, in order for the GUP and CG predictions to be consistent, $\beta$ must be of order unity.

Furthermore, we have speculated on the sign of $\beta$. Although on this matter we are still far from the definitive 
solution, a preliminary analytic evaluation of the evaporation rate within the framework of Horizon Quantum Mechanics and some considerations related to the conservation of energy, suggest that the most plausible picture is the one with a negative deformation parameter, $\beta<0$.

In this connection, we emphasize that a similar result would not be surprising in the context of a corpuscular (i.e discrete) description of black holes; in Ref. [29], indeed, it was shown that a GUP with $\beta<0$ can be derived assuming that the universe has an underlying crystal lattice-like structure. In Sect. 2 we have also seen that, in the case $\beta>0$, black holes would not entirely evaporate. However, if on the one hand black hole remnants may be viewed as potential candidates for dark matter [54,55], on the other hand their existence would be rather problematic for the entropy/information paradox [48].

Apart from these supporting arguments, it also worth remarking that, if $\beta<0$, then from Eq. (3) there would be a maximum value of $\delta p$ around the Planck scale for which $\delta x \delta p=0$, i.e. the quantum uncertainty would be completely erased. Therefore, it would seem that physics can become classical again at Planck scale. The possibility of a classical Planckian regime has been already discussed in Ref. [29] in connection with the doubly special relativity model [56-59] and with the deterministic interpretation of quantum mechanics [60]. Further arguments have been given in Ref. [61] by regarding $\hbar$ as a dynamical field that vanishes in the Planckian limit.

Given the absolute lack of knowledge about physics at Planck scale, it is clear that in principle all possible scenarios should be contemplated in order to achieve a better understanding of how gravity and quantum effects behave when combined together. For example, one cannot exclude a priori the possibility that $\beta$ is a function rather than a pure number. This has been originally suggested in Ref. [62] as condition for black hole complementarity principle to always hold. A similar result has been recently recovered in Ref. [23], where, by conjecturing the equality between the GUPdeformed black hole temperature of a Schwarzschild black hole and the modified Hawking temperature of a quantumcorrected Schwarzschild black hole, it has been obtained a GUP parameter depending on the ratio $m_{p} / M$.

In light of the above discussion, the present investigation should thus be regarded as a further attempt to gain information about the GUP black hole physics through a connection with an intrinsically corpuscular description of gravity. More work is inevitably required to provide a definite answer about the sign of the GUP deformation parameter $\beta$.

Acknowledgements The authors would like to thank Roberto Casadio and Andrea Giusti for helpful conversations.

Data Availability Statement This manuscript has no associated data or the data will not be deposited. [Authors' comment: Data sharing not applicable to this article as no datasets were generated or analyzed during the current study.]

Open Access This article is distributed under the terms of the Creative Commons Attribution 4.0 International License (http://creativecomm ons.org/licenses/by/4.0/), which permits unrestricted use, distribution, and reproduction in any medium, provided you give appropriate credit to the original author(s) and the source, provide a link to the Creative Commons license, and indicate if changes were made.

Funded by SCOAP ${ }^{3}$.

\section{References}

1. W. Heisenberg, Z. Phys. 43, 172 (1927)

2. M. Maggiore, Phys. Rev. D 49, 5182 (1994)

3. J.A. Wheeler, Phys. Rev. 97, 511 (1955)

4. H.S. Snyder, Phys. Rev. 71, 38 (1947)

5. C.N. Yang, Phys. Rev. 72, 874 (1947)

6. C.A. Mead, Phys. Rev. 135, B849 (1964)

7. F. Karolyhazy, Nuovo Cim. A 42,390 (1966)

8. D. Amati, M. Ciafaloni, G. Veneziano, Phys. Lett. B 197, 81 (1987)

9. D.J. Gross, P.F. Mende, Phys. Lett. B 197, 129 (1987)

10. D. Amati, M. Ciafaloni, G. Veneziano, Phys. Lett. B 216, 41 (1989)

11. K. Konishi, G. Paffuti, P. Provero, Phys. Lett. B 234, 276 (1990)

12. M. Maggiore, Phys. Lett. B 319, 83 (1993)

13. F. Scardigli, Nuovo Cim. B 110, 1029 (1995)

14. F. Scardigli, Phys. Lett. B 452, 39 (1999)

15. S. Capozziello, G. Lambiase, G. Scarpetta, Int. J. Theor. Phys. 39, $15(2000)$

16. M. Bojowald, A. Kempf, Phys. Rev. D 86, 085017 (2012)

17. F. Scardigli, R. Casadio, Class. Quant. Grav. 20, 3915 (2003)

18. R.J. Adler, D.I. Santiago, Mod. Phys. Lett. A 14, 1371 (1999)

19. R.J. Adler, P. Chen, D.I. Santiago, Gen. Rel. Grav. 33, 2101 (2001)

20. R. Casadio, R. Garattini, F. Scardigli, Phys. Lett. B 679, 156 (2009)

21. R. Casadio, F. Scardigli, Eur. Phys. J. C 74, 2685 (2014)

22. F. Scardigli, G. Lambiase, E. Vagenas, Phys. Lett. B 767, 242 (2017)

23. E.C. Vagenas, S.M. Alsaleh, A. Farag, EPL 120, 40001 (2017)

24. G. Lambiase, F. Scardigli, Phys. Rev. D 97, 075003 (2018)

25. F. Scardigli, M. Blasone, G. Luciano, R. Casadio, Eur. Phys. J. C 78, 728 (2018)

26. G.G. Luciano, L. Petruzziello, Eur. Phys. J. C 79, 283 (2019)

27. M. Blasone, G. Lambiase, G.G. Luciano, L. Petruzziello, F. Scardigli. arXiv:1902.02414 [hep-th]

28. T. Kanazawa, G. Lambiase, G. Vilasi, A. Yoshioka, Eur. Phys. J. C 79, 95 (2019)

29. P. Jizba, H. Kleinert, F. Scardigli, Phys. Rev. D 81, 084030 (2010)

30. Y.C. Ong, JCAP 1809, 015 (2018)

31. Y.C. Ong, Y. Yao, Phys. Rev. D 98, 126018 (2018)

32. F. Scardigli, R. Casadio, Eur. Phys. J. C 75, 425 (2015)

33. G. Dvali, C. Gomez, Fortsch. Phys. 61, 742 (2013)

34. G. Dvali, C. Gomez, Eur. Phys. J. C 74, 2752 (2014)

35. R. Casadio, A. Orlandi, JHEP 1308, 025 (2013)

36. R. Casadio, A. Giugno, O. Micu, A. Orlandi, Phys. Rev. D 90, $084040(2014)$

37. G. Dvali, D. Flassig, C. Gomez, A. Pritzel, N. Wintergerst, Phys. Rev. D 88, 124041 (2013)

38. G. Dvali, C. Gomez, JCAP 1401, 023 (2014)

39. G. Dvali, C. Gomez, R.S. Isermann, D. Lüst, S. Stieberger, Nucl. Phys. B 893, 187 (2015)

40. G. Dvali, Fortsch. Phys. 64, 106 (2016)

41. R. Casadio, A. Giugno, A. Orlandi, Phys. Rev. D 91, 124069 (2015)

42. R. Casadio, A. Giugno, A. Giusti, Phys. Lett. B 763, 337 (2016)

43. R. Casadio, R. da Rocha, Phys. Lett. B 763, 434 (2016) 
44. D.N. Page, Phys. Rev. D 13, 198 (1976)

45. P. Chen, Y.C. Ong, Dh Yeom, Phys. Rept 603, 1 (2015)

46. Y. Aharonov, A. Casher, S. Nussinov, Phys. Lett. B 191, 51 (1987)

47. S.D. Mathur, Class. Quant. Grav. 26, 224001 (2009)

48. J.D. Bekenstein, Phys. Rev. D 49, 1912 (1994)

49. N.N. Bogolyubov, J. Phys. (USSR) 11, 23 (1947) [Izv. Akad. Nauk Ser. Fiz. 11, 77 (1947)]

50. R. Casadio. arXiv:1305.3195 [gr-qc]

51. R. Casadio, A. Giugno, A. Giusti, Gen. Rel. Grav. 49, 32 (2017)

52. R. Casadio, B. Harms, Y. Leblanc, Phys. Rev. D 58, 044014 (1998)

53. G.L. Alberghi, R. Casadio, A. Tronconi, J. Phys. G 34, 767 (2007)

54. P. Chen, Mod. Phys. Lett. A 19, 1047 (2004)
55. P. Chen, New Astron. Rev 49, 233 (2005)

56. G. Amelino-Camelia, Int. J. Mod. Phys. D 11, 35 (2002)

57. J. Magueijo, L. Smolin, Phys. Rev. Lett. 88, 190403 (2002)

58. G. Amelino-Camelia, Nature 418, 34 (2002)

59. J. Magueijo, L. Smolin, Phys. Rev. D 67, 044017 (2003)

60. G. 't Hooft, The cellular automaton interpretation of quantum mechanics. Fundam. Theor. Phys. 185, 49-55 (2006)

61. S. Hossenfelder, Phys. Lett. B 725, 473 (2013)

62. P. Chen, Y.C. Ong, Dh Yeom, JHEP 1412, 021 (2014) 